University of Wollongong

Research Online

Faculty of Engineering and Information

Faculty of Engineering and Information

Sciences - Papers: Part B

Sciences

2017

\title{
Double-Mode Microparticle Manipulation by Tunable Secondary Flow in Microchannel With Arc-Shaped Groove Arrays
}

Qianbin Zhao

University of Wollongong, qz260@uowmail.edu.au

Sheng Yan

University of Wollongong, sy034@uowmail.edu.au

Dan Yuan

University of Wollongong, dy983@uowmail.edu.au

Jun Zhang

Nanjing University of Science and Technology

Haiping Du

University of Wollongong, hdu@uow.edu.au

See next page for additional authors

Follow this and additional works at: https://ro.uow.edu.au/eispapers1

Part of the Engineering Commons, and the Science and Technology Studies Commons

Research Online is the open access institutional repository for the University of Wollongong. For further information contact the UOW Library: research-pubs@uow.edu.au 


\title{
Double-Mode Microparticle Manipulation by Tunable Secondary Flow in Microchannel With Arc-Shaped Groove Arrays
}

\author{
Abstract \\ In this paper, we proposed a microparticle manipulation approach, by which particles are able to be \\ guided to different equilibrium positions through modulating the Reynolds number. In the microchannel \\ with arc-shaped groove arrays, secondary flow vortex arisen due to the pressure gradient varies in the \\ aspects of both magnitude and shape with the increase of Reynolds number. And the variation of \\ secondary flow vortex brings about different focusing modes of microparticles in the microchannel. We \\ investigated the focusing phenomenon experimentally and analyzed the mechanism through numerical \\ simulations. At a high Reynolds number (Re \& \#x003D; 127.27), the geometry-induced secondary flow \\ rotates constantly along a direction, and most particles are guided to the equilibrium position near one \\ side of the microchannel. However, at a low Reynolds number (Re \& \#x003D; 2.39), the shapes of \\ geometry-induced secondary flow vortices are obviously different, forming a variant Dean-like vortex that \\ consists of two asymmetric counter-rotating streams in cross sections of the straight channel. Because \\ of the periodical effects, suspended particles are concentrated at another equilibrium position on the \\ opposite side of the microchannel. Meanwhile, the effects of particle size influence both the focusing \\ position and quality in regimes.

\section{Disciplines} \\ Engineering | Science and Technology Studies

\section{Publication Details} \\ Zhao, Q., Yan, S., Yuan, D., Zhang, J., Du, H., Alici, G. \& Li, W. (2017). Double-Mode Microparticle \\ Manipulation by Tunable Secondary Flow in Microchannel With Arc-Shaped Groove Arrays. IEEE \\ Transactions on Biomedical Circuits and Systems, 11 (6), 1406-1412.

\section{Authors} \\ Qianbin Zhao, Sheng Yan, Dan Yuan, Jun Zhang, Haiping Du, Gursel Alici, and Weihua Li
}




\title{
Double-mode Microparticle Manipulation by Tunable Secondary Flow in Microchannel with Arc-shaped Groove Arrays
}

\author{
Qianbin Zhao, Sheng Yan, Dan Yuan, Jun Zhang, Member, IEEE, Haiping Du, Member, IEEE, Gursel \\ Alici1, Member, IEEE, and Weihua Li, Member, IEEE
}

\begin{abstract}
In this paper, we proposed a microparticle manipulation approach, by which particles are able to be guided to different equilibrium positions through modulating the Reynolds number. In the microchannel with arc-shaped groove arrays, secondary flow vortex arisen due to the pressure gradient varies in the aspects of both magnitude and shape with the increase of Reynolds number. And the variation of secondary flow vortex brings about different focusing modes of microparticles in the microchannel. We investigated the focusing phenomenon experimentally and analyzed the mechanism through numerical simulations. At a high Reynolds number $(\operatorname{Re}=127.27)$, the geometry-induced secondary flow rotates constantly along a direction, and most particles are guided to the equilibrium position near one side of the microchannel. However, at a low Reynolds number $(\operatorname{Re}=2.39)$, the shapes of geometry-induced secondary flow vortices are obviously different, forming a variant Dean-like vortex which consists of two asymmetric counter-rotating streams in cross sections of the straight channel. Because of the periodical effects, suspended particles are concentrated at another equilibrium position on the opposite side of the microchannel. Meanwhile, the effects of particle size influence both the focusing position and quality in regimes.
\end{abstract}

Index Terms-Arc-shaped groove arrays, inertial focusing, microfluidics, microparticle manipulation, secondary flow.

\section{INTRODUCTION}

M ICROFLUIDICS has made significant contributions to the fields of disease diagnostics and biological assays [1-3]. Sample preparation is an indispensable component in many microfluidic point-of-care (POC) diagnostic systems [4]. Sample preparation can be accomplished using simple porous fiber strips in some applications, such as diagnosis of blood glucose $[5,6]$. One example is the recently developed smartphone based POC diagnosis devices, which have been

This work was supported in part by the University of Wollongong -China Scholarship Council joint scholarships.

Qianbin Zhao, Sheng Yan, Dan Yuan, Gursel Alici1 and Weihua Li are now with the School of Mechanical, Materials, Mechatronic and Biomedical Engineering, University of Wollongong, Wollongong, NSW 2522, Australia (e-mail: weihuali@uow.edu.au).

Jun Zhang is with the School of Mechanical Engineering, Nanjing University of Science and Technology, Nanjing 210094, China (e-mail: junzhang@njust.edu.cn).

Haiping Du is with the School of Electric, Computer and Telecommunication Engineering, University of Wollongong, Wollongong, NSW 2522, Australia. contributed significantly by the groups of Guo [7, 8] and Hall [9]. In these devices, the fiber strip extracts the blood plasma to the region of sensor through the intrinsic capillary force. However, in most applications, sample preparation requires much more complex processes $[10,11]$. Therefore, development of advanced microfluidic manipulation (such as particle focusing, ordering and separation etc.) technique is of great importance.

Inertial microfluidics has been one of the most popular microfluidic techniques in the application of microparticle manipulation recent years, such as microparticle focusing [12] and separation [13]. Inertial microfluidic technique is a label-free passive manipulation method, which relies on the interaction of particles and fluids in the inertial flow $[14,15]$. In the microfluidics, flows are regarded as laminar because the Reynolds number is far less than 2000 in typical cases [16]. And inertial microfluidics works in the middle Reynolds number range (typically from $\sim 1$ to $\sim 100$ ) between Stokes flow and turbulent flows, where the inertia and fluid viscosity are finite $[14,17]$.

In the regime of inertial microfluidics, two dominating inertial effects, which are induced by the finite inertia of flow, are widely employed to manipulate microparticles: (1) inertial migration and (2) secondary flow. Inertial migration is the phenomenon that randomly distributed particles migrate to several equilibrium positions when traveling in a straight channel $[18,19]$. And it has been demonstrated that, in a straight channel with the square cross section, four equilibrium positions form centered at the faces of the channel at a modulated Reynolds number [20]. However, another fact has also been demonstrated that the number of equilibrium position is closely related to the aspect ratio (AR, defined as the ratio of channel height to channel width) of channel, for example, there are two equilibrium positions forming in the straight channel with low AR cross section ( $\mathrm{AR}=0.5)$ [21-23].

Secondary flow in inertial microfluidics is typically generated in the curved structure [24] or disturbance obstacle structure $[23,25,26]$ within the microchannel because of the pressure gradient perpendicular to the main flow direction. In a curved channel, the mismatch of fluid momentum in the center and near-wall region induces two counter-rotating secondary flow vortices, which is regarded as Dean vortex [14, 17]. Meanwhile, it has been reported that the shape of secondary 
flow is associated with the Dean number $\kappa$ in microchannel. With the increase of Dean number, the center of the two symmetric vortices is going to move outwards to the outer channel wall in the radial direction [24]. And this dimensionless parameter $\kappa$ was proposed by Berger et al., expressed as [24]:

$$
\kappa=\left(\frac{H}{2 R}\right)^{1 / 2} \operatorname{Re}
$$

where, $H$ and $R$ are the hydraulic diameter of channel and radius of curvature, respectively.

Various types of channels have been designed to employ different secondary flows to manipulate suspended particles, such as the serpentine [12, 27], spiral [28, 29] and contraction-expansion array (CEA) channel [22]. Besides employing one layer channel to generate Dean vortex, the double layer microchannel, which is fabricated by the two-step photolithography technique, has also been used to take advantage of symmetric and asymmetric secondary flow for microparticle manipulation [23,30].

The herringbone and slanted groove channel were first employed as the micromixer through generating transverse flows in microchannels by a steady axial pressure gradient [31, 32]. Moreover, Di Carlo et al. proposed the microvortex manipulation (MVM) approach to guide particles using the interaction of hydrodynamic, gravitational and buoyant force. And a mixture containing binary particles with a density difference was successfully sorted using the device [30]. In addition, a straight low aspect ratio channel with orthogonally arranged step arrays was demonstrated to focus particle in a single-stream without sheath fluids or external force using symmetric geometry-induced secondary flow vortices, which replaced the two original inertial migration equilibrium positions [23].

At a low Reynolds number (in the typical microfluidic system), the drag force of geometry-induced secondary flow exerting on the small rigid spherical particle can be expressed as:

$$
F_{D}=6 \pi a \eta\left(v_{f}-v_{p}\right)
$$

where $a$ is the radius of the particle, $\eta$ is the viscosity of the fluid, $v_{f}$ is the dynamic velocity of the fluid, and $v_{p}$ is the velocity of the particle [33].

In this work, we will demonstrate that the microchannel with arc-shaped groove arrays has two regimes at high and low Reynolds number respectively, in which the same particles will be guided into different equilibrium positions (Fig. 1(a)). When applied with a high flow rate, particles are concentrated qualifiedly near the sidewall and center of the channel depending on the particle size. When applied with a low flow rate, different-size particles are concentrated at another equilibrium position near the opposite sidewall. And the effects of particle size are critical for the particle focusing performance. Large particles were observed to concentrate as a narrower streamline than the small ones. Numerical simulations of flow field were conducted to analyze and explain the multiple microparticle focusing phenomenon. Different secondary flow vortices are induced because of the flow field disturbance by the expansive groove structure. As a result, the corresponding drag force would guide the particles into their equilibrium positions and balanced with the inertial lift force simultaneously. This work reports a microchannel to manipulate microparticles into different designated equilibrium positions by taking advantage of modulating the applied flow rate (Reynolds number).

\section{EXPERIMENTS AND MATERIALS}

\section{A. Microchannel}

The microchannel used in this work is a stacked structure composed of a straight channel $(\mathrm{AR}=0.1)$ and 50 arc-shaped groove arrays (Fig. 1(b)). For the straight channel, the cross section is a $200 \times 20 \mu \mathrm{m}$ (width $(W) \times$ height $(H))$ rectangle. 50 arc-shaped grooves are arranged with $50 \mu \mathrm{m}$ spacing $\left(H_{\mathrm{s}}\right)$ on the top of the straight channel. Each groove pattern is arc-shaped with a small curvature of $600 \mu \mathrm{m}\left(R_{1}\right)$ and a large curvature of $650 \mu \mathrm{m}\left(R_{2}\right)$. And the height of the expansive groove structure $\left(H_{\mathrm{g}}\right)$ is $20 \mu \mathrm{m}$. The details of similar microchannel fabrication have been reported in our previous work.[34]

\section{B. Fluorescent microparticle suspensions}

The commercial fluorescent polystyrene microparticles were purchased from Thermo Fisher Scientific. $13 \mu \mathrm{m}$ (CAT. NO. $36-4,16 \% \mathrm{CV}$ ) and $8 \mu \mathrm{m}$ particles (CAT. NO. 36-3, 18\%CV) are in red fluorescence, while $4.8 \mu \mathrm{m}$ particles (CAT. NO. $\mathrm{G} 0500,5 \% \mathrm{CV})$ are green fluorescent. Microparticles were suspended in deionized (DI) water, and $0.1 \% \mathrm{w} / \mathrm{v}$ Tween 20 (Sigma-Aldrich, Product NO. P9416) was added to prevent the particle aggregation and adhesion to the channel walls.

\section{Experiment setup}

The microchannel was placed on an inverted microscope (CKX41, Olympus, Japan). Particle suspensions were injected into the microchannel through a syringe pump (Legato $100, \mathrm{Kd}$ Scientific) at different flow rates. And a mercury arc lamp was used to illuminate the particle trajectories within the channel for observation and recording. A CCD camera (Optimos, Q-imaging, Australia) was used to capture the fluorescent images with a $15 \mathrm{~ms}$ exposure time. All images were then processed and analyzed using the image processing software (Q-Capture Pro 7, Q-imaging, Australia). And all fluorescent images were obtained by stacking 50 consecutive frames in order to analyze particle trajectory patterns.

\section{EXPERIMENT RESULTS}

\section{A. AT high Reynolds number}

The full width at half maximum (FWHM) of fluorescent streaks is widely adopted to assess the particle focusing performance. In this work, we used this approach to measure the width and lateral position of the particle trajectory in the normalized intensity profiles. According to this assessment approach, if the measured FWHM was less than 2 times the diameter of focused particle, it is able to be considered as a 
qualified particle focusing [35].

At a high Reynolds number $(\mathrm{Re}=127.27)$, particles were able to be concentrated in the microchannel. The optical images (Fig. 2(a)) show that large particles (13 and $8 \mu \mathrm{m}$ in diameter) were concentrated near the sidewall 1 of the channel, while the small particles $(4.8 \mu \mathrm{m}$ in diameter) were mostly concentrated in a narrow fluid stream between the sidewall 2 and center of the channel. First, we conducted an experiment on the high concentration $13 \mu \mathrm{m}$ particle suspensions $\left(\sim 10^{6}\right.$ counts $\left./ \mathrm{ml}\right)$. The fluorescent image shows that all particles were concentrated well at the equilibrium position, however, the FWHM measured (Fig. 2(b)) was more than $26 \mu \mathrm{m}$ (2 times the diameter of $13 \mu \mathrm{m}$ particle). Next, another experiment on the relatively low concentration particle suspensions $\left(\sim 8 \times 10^{4}\right.$ counts/ml) was conducted, and the FWHM measured was narrower and less than $26 \mu \mathrm{m}$. Besides, $8 \mu \mathrm{m}$ and $4.8 \mu \mathrm{m}$ particles were also able to be concentrated in the microchannel and the FWHM of them were narrow enough as well (Fig. 2(b)). But the focusing performances of these smaller particles were not as good as $13 \mu \mathrm{m}$ particles shown as the normalized intensity profiles. Especially, it could be still found that a few of 4.8 particles were unfocused and distributed randomly in the microchannel. Apparent particle trajectory pattern could be also observed within the grooves.

\section{B. AT low Reynolds number}

At the low Reynolds number $(\operatorname{Re}=2.39)$, the trajectory patterns of particles in microchannel were obviously different from those at the high Reynolds number. In the optical images (Fig. 3(a)), it is shown that particles were located at another equilibrium position near the sidewall 2. And the FWHM of different-size particles measured in the normalized intensity profiles (Fig. 3(b)) were various distinctly because of the effects of particle size. The particle focusing performance (particle trajectory pattern distribution) was proportional to the particle size. Applied with the same flow rate $(15 \mu 1 / \mathrm{min}), 13$ $\mu \mathrm{m}$ particles could reach a stable qualified focusing condition as its FWHM was narrow enough, while $8 \mu \mathrm{m}$ particles and 4.8 $\mu \mathrm{m}$ particles were distributed as the wide trajectory streams. In addition, compared with the particle distribution at the high Reynolds number, particles as small as $4.8 \mu \mathrm{m}$ could still retain in the straight channel without being trapped into the expansive groove structure. It indicates that although the particle trajectory (4.8 $\mu \mathrm{m}$ particle) was very wide, no randomly distributed particles were observed within the channel. Meanwhile, the particle trajectory $(13 \mu \mathrm{m})$ patterns between the high (127.27) and low Reynolds number (2.39) are captured and provided in Fig. 4. It could be found that the equilibrium position of $13 \mu \mathrm{m}$ particle near sidewall 2 was disturbed and disappeared when the Reynolds number exceeded 2.39, and the particle trajectory were gradually pulled to the sidewall 1 with the increase of Reynolds number.

\section{DISCUSSION}

In order to analyze the mechanism of this double-mode particle focusing, the flow field of the grooved channel was simulated through COMSOL at a high Reynolds number
$(\operatorname{Re}=127.27)$ and a low Reynolds number $(\operatorname{Re}=2.39)$, respectively. Because the microchannel used in the experiments consists of a straight channel and 50 arc-shaped groove arrays, a segment of the channel with one arc-shaped groove structure pattern was built and imported to save the computational sources and time. The 3D model of the channel segment was divided into 4 cross-sectional plots to calculate and visualize the geometry-induced secondary flow vortices in the transverse direction. It could be observed that the disturbing influences on the flow fields were significantly different between two Reynolds number conditions. More details about the simulation are provided in the supplementary material.

From the simulation results of flow field at the high Reynolds number (Fig. 5(a)), the streamline analysis of typical transverse flow velocity profile within cross sections depicts the formation and development of a complete oval microvortex induced by the expansive arc-shaped groove structure on the top surface of the microchannel. The secondary flow vortex constantly points to $-Z$ direction in the bottom part of channel cross sections, while pointing to the opposite direction $(Z)$ in the expansive groove part, together forming a stable complete clockwise circulation. As a result, the corresponding drag force could guide particles to the stable equilibrium position (the green box). Large particles ( 13 and $8 \mu \mathrm{m}$ ) would be pulled by a strong drag force (refer to (2)) to the direction of $-\mathrm{Z}$ and settle near the sidewall 1 , balanced by the inertial lift force along the vertical direction. Because the secondary flow and inertial lift force are both proportional to particle sizes, large $(13 \mu \mathrm{m})$ particles can be focused more tightly than the smaller $(8 \mu \mathrm{m})$ ones, exhibiting a better focusing performance in the experiments. With regard to $4.8 \mu \mathrm{m}$ particles, they were not able to be pushed and focused to the sidewall 1 where large size particles $(13$ and $8 \mu \mathrm{m})$ settled. Most of them were trapped in the core of the microvotex, where the magnitude of secondary flow on $\mathrm{Z}$ direction is minimum. And some $4.8 \mu \mathrm{m}$ particles were forced to follow the vortex and trapped into the groove structure following the circulating streams, resulting in some randomly distributed particles within the channel.

From the simulation results of the flow field at the low Reynolds number (Fig. 5(b)), the shapes of geometry-induced secondary flow vortices are significantly different from that at the high Reynolds number, as there is no apparent oval microvortex could be made out. Compared with the unidirectional circulating geometry-induced secondary flow vortex at the high Reynolds number, the cross-sectional flow field at the low Reynolds number was more similar to a variant Dean-like vortex, and two counter-rotating vortices seems to be formed (plot (i) and (ii) in Figure 5(b) and supplementary material Fig. S2). The shapes of vortices changes along the cross-sectional horizontal direction. The left vortex (near sidewall 1) was expanding, while the right one (near sidewall 2) was contracting conversely. As a result, the center of two counter-rotating vortices migrated from left to right (more simulated cross-sectional plots are provided in the supplementary material, which depicts the development of two microvortices clearly and in detail). This periodical development could be induced by each arc-shaped groove structure, and this double-vortex interaction could gradually 
guide particles to the right sidewall 2 following the migration of the center of counter-rotating vortices. Therefore, a dynamic equilibrium position was formed, which agreed with the experiment results. Moreover, a stronger secondary flow drag force exerts on larger particles and stabilize the particle focusing, therefore $13 \mu \mathrm{m}$ particle trajectories were pinched within a narrower streak than those of smaller ones $(8$ and 4.8 $\mu \mathrm{m}$ particles). Meanwhile, the vertical component of vortex drag force, which tends to pull particles into the groove structures, is much weaker compared with that at the high Reynolds number. As a result, small size particles $(4.8 \mu \mathrm{m})$ could still retain within the bottom part of the straight channel, not being trapped into expansive groove structure. In conclusion, the change of geometry-induced secondary flow vortex shape due to variation of Reynolds numbers results in the distinct particle focusing modes and different equilibrium positions in the channel with arc-shaped groove arrays.

\section{REFERENCES}

[1] A. A. Adams, P. I. Okagbare, J. Feng, M. L. Hupert, D. Patterson, J. Göttert, et al., "Highly efficient circulating tumor cell isolation from whole blood and label-free enumeration using polymer-based microfluidics with an integrated conductivity sensor," Journal of the American Chemical Society, vol. 130, pp. 8633-8641, 2008.

[2] K. Hoshino, Y.-Y. Huang, N. Lane, M. Huebschman, J. W. Uhr, E. P. Frenkel, et al., "Microchip-based immunomagnetic detection of circulating tumor cells," Lab on a Chip, vol. 11, pp. 3449-3457, 2011.

[3] S. Nagrath, L. V. Sequist, S. Maheswaran, D. W. Bell, D. Irimia, L. Ulkus, et al., "Isolation of rare circulating tumour cells in cancer patients by microchip technology," Nature, vol. 450, pp. 1235-1239, 2007.

[4] C. D. Chin, V. Linder, and S. K. Sia, "Lab-on-a-chip devices for global health: past studies and future opportunities," Lab on A Chip, vol. 7, pp. 41-57, 2007.

[5] A. K. Yetisen, M. S. Akram, and C. R. Lowe, "Paper-based microfluidic point-of-care diagnostic devices," Lab on A Chip, vol. 13, pp. 2210-51, 2013.

[6] J. Yu, L. Ge, J. Huang, S. Wang, and S. Ge, "Microfluidic paper-based chemiluminescence biosensor for simultaneous determination of glucose and uric acid," Lab on A Chip, vol. 11, p. 1286,2011

[7] J. Guo and X. Ma, "Simultaneous monitoring of glucose and uric acid on a single test strip with dual channels," Biosensors and Bioelectronics, vol. 94, pp. 415-419, 2017.

[8] J. Guo, "Uric Acid Monitoring with Smartphone as Electrochemical Analyzer," Analytical Chemistry, 2016.

[9] A. C. Sun, C. Yao, A. G. Venkatesh, and D. A. Hall, "An efficient power harvesting mobile phone-based electrochemical biosensor for point-of-care health monitoring," Sensors \& Actuators B Chemical, vol. 235, pp. 126-135, 2016.

[10] A. J. Mach, O. B. Adeyiga, and D. Di Carlo, "Microfluidic sample preparation for diagnostic cytopathology," Lab on a chip, vol. 13, pp. 1011-1026, 2013.

[11] M. Kersaudy-Kerhoas and E. Sollier, "Micro-scale blood plasma separation: from acoustophoresis to egg-beaters," Lab on a Chip, vol. 13, pp. 3323-3346, 2013.

[12] D. Di Carlo, D. Irimia, R. G. Tompkins, and M. Toner, "Continuous inertial focusing, ordering, and separation of particles in microchannels," Proceedings of the National Academy of Sciences, vol. 104, pp. 18892-18897, 2007.

[13] M. E. Warkiani, G. Guan, K. B. Luan, W. C. Lee, A. A. S. Bhagat, P. K. Chaudhuri, et al., "Slanted spiral microfluidics for the ultra-fast, label-free isolation of circulating tumor cells," Lab on a Chip, vol. 14, pp. 128-137, 2014.

[14] D. Di Carlo, "Inertial microfluidics," Lab on a Chip, vol. 9, pp. 3038-3046, 2009.
[15] S. C. Hur, T. Z. Brinckerhoff, C. M. Walthers, J. C. Dunn, and D. Di Carlo, "Label-free enrichment of adrenal cortical progenitor cells using inertial microfluidics," PloS one, vol. 7, p. e46550, 2012.

[16] T. M. Squires and S. R. Quake, "Microfluidics: Fluid physics at the nanoliter scale," Reviews of modern physics, vol. 77, p. 977, 2005.

[17] J. Zhang, S. Yan, D. Yuan, G. Alici, N.-T. Nguyen, M. E. Warkiani, et al., "Fundamentals and applications of inertial microfluidics: a review," Lab on a Chip, vol. 16, pp. 10-34, 2016.

[18] G. Segre, "Radial particle displacements in Poiseuille flow of suspensions," Nature, vol. 189, pp. 209-210, 1961.

[19] G. Segre and A. Silberberg, "Behaviour of macroscopic rigid spheres in Poiseuille flow Part 2. Experimental results and interpretation," Journal of Fluid Mechanics, vol. 14, pp. 136-157, 1962.

[20] Y.-S. Choi, K.-W. Seo, and S.-J. Lee, "Lateral and cross-lateral focusing of spherical particles in a square microchannel," Lab on a Chip, vol. 11, pp. 460-465, 2011.

[21] J. Zhou and I. Papautsky, "Fundamentals of inertial focusing in microchannels," Lab on a Chip, vol. 13, pp. 1121-1132, 2013.

[22] M. G. Lee, S. Choi, and J.-K. Park, "Inertial separation in a contraction-expansion array microchannel," Journal of Chromatography A, vol. 1218, pp. 4138-4143, 2011.

[23] A. J. Chung, D. R. Gossett, and D. Di Carlo, "Three Dimensional, Sheathless, and High - Throughput Microparticle Inertial Focusing Through Geometry - Induced Secondary Flows," Small, vol. 9, pp. 685-690, 2013.

[24] S. Berger, L. Talbot, and L. Yao, "Flow in curved pipes," Annual review of fluid mechanics, vol. 15, pp. 461-512, 1983.

[25] H. Amini, E. Sollier, M. Masaeli, Y. Xie, B. Ganapathysubramanian, H. A. Stone, et al., "Engineering fluid flow using sequenced microstructures," Nature communications, vol. 4, p. $1826,2013$.

[26] Q. Zhao, D. Yuan, S. Yan, J. Zhang, H. Du, G. Alici, et al., "Flow rate-insensitive microparticle separation and filtration using a microchannel with arc-shaped groove arrays," Microfluidics and Nanofluidics, vol. 21, p. 55, 2017.

[27] J. Zhang, S. Yan, W. Li, G. Alici, and N.-T. Nguyen, "High throughput extraction of plasma using a secondary flow-aided inertial microfluidic device," RSC Advances, vol. 4, pp. 33149-33159, 2014.

[28] A. A. S. Bhagat, S. S. Kuntaegowdanahalli, and I. Papautsky, "Continuous particle separation in spiral microchannels using dean flows and differential migration," Lab on a Chip, vol. 8, pp. 1906-1914, 2008.

[29] J. Sun, M. Li, C. Liu, Y. Zhang, D. Liu, W. Liu, et al., "Double spiral microchannel for label-free tumor cell separation and enrichment," Lab on a chip, vol. 12, pp. 3952-3960, 2012.

[30] C.-H. Hsu, D. Di Carlo, C. Chen, D. Irimia, and M. Toner, "Microvortex for focusing, guiding and sorting of particles," Lab on a Chip, vol. 8, pp. 2128-2134, 2008.

[31] A. D. Stroock, S. K. Dertinger, A. Ajdari, I. Mezić, H. A. Stone, and G. M. Whitesides, "Chaotic mixer for microchannels," Science, vol. 295, pp. 647-651, 2002.

[32] N. S. Lynn and D. S. Dandy, "Geometrical optimization of helical flow in grooved micromixers," Lab on a Chip, vol. 7, pp. 580-587, 2007.

[33] T. Gerlach, "Microdiffusers as dynamic passive valves for micropump applications," Sensors and Actuators A: Physical, vol. 69, pp. 181-191, 1998.

[34] Q. Zhao, J. Zhang, S. Yan, D. Yuan, H. Du, G. Alici, et al., "High-throughput sheathless and three-dimensional microparticle focusing using a microchannel with arc-shaped groove arrays," Scientific Reports, vol. 7, 2017.

[35] J. M. Martel and M. Toner, "Inertial focusing dynamics in spiral microchannels," physics of fluids, vol. 24, p. 032001, 2012.

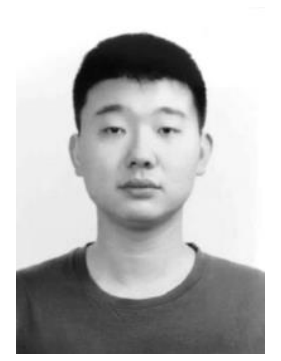

Qianbin Zhao received his Bachelor degree in engineering from the Northeastern University (NEU), Shenyang, China, in 2015. He recently holds a position as a $\mathrm{PhD}$ candidate in 
the School of Mechanical, Materials, Mechatronic and Biomedical Engineering at University of Wollongong. His current research focuses on (i) inertial microfluidics for microparticle manipulation through geometry-induced secondary flow and (ii) viscoelastic microfluidics applications for bioassays and biosample processing.

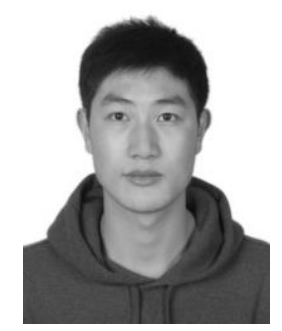

Sheng Yan received his Bachelor degree of Engineering with an Outstanding Graduate award from China University of Mining and Technology in 2012 and doctoral degree from University of Wollongong in 2016. He currently works as an associate research fellow in the School of Mechanical, Materials and Mechatronic Engineering at University of Wollongong. His main research focus is to develop hybrid techniques to tune hydrophoresis for cell focusing and separation. His research interests include dielectrophoresis, hydrophoresis, magnetophoresis, neurosciences, computational fluid dynamics (CFD).

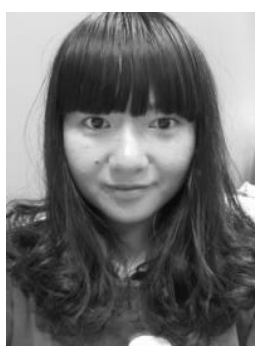

Dan Yuan was born in Shandong province, China, in 1990. She received her Bachelor degree of Engineering and Master degrees of Engineering from Hefei University of Technology in Hefei, China, in 2011 and 2014, and majored in Precision Measurement Technology and Instruments.

Since 2014, she has been a PhD candidate in the School of Mechanical, Materials, Mechatronic and Biomedical Engineering at University of Wollongong. She is the author of more than 18 papers. Her research interests are particle and cell manipulation in viscoelastic fluid.

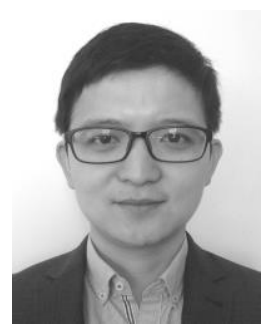

Jun Zhang received his bachelor degree in Engineering from the Nanjing University of Science and Technology (NJUST), Nanjing, China in 2009 with an Outstanding Graduate award and received a $\mathrm{PhD}$ degree in Mechanical Engineering from University of Wollongong, Australia in 2015.

After that, he conducted his postdoctoral research as a research scientist and an associate research fellow at Woolcock Institute of Medical Research and University of Wollongong, respectively. He is currently an Associate Professor with Nanjing University of Science and Technology (NJUST), China. He has published more than 30 journal and conference articles.

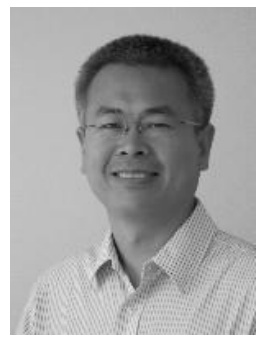

Haiping Du (M'09-SM'17) received the Ph.D. degree in mechanical design and theory from Shanghai Jiao Tong University, Shanghai, China, in 2002. He was a Research Fellow with the University of Technology, Sydney, from 2005 to 2009, and was a Postdoctoral Research Associate with Imperial College London from 2004 to 2005 and the University of Hong Kong from 2002 to 2003. He is currently a Professor at the School of Electrical, Computer and Telecommunications Engineering, University of Wollongong, Wollongong, N.S.W, Australia. He is a Subject Editor of the Journal of Franklin Institute and an Associate Editor of IEEE Control Systems Society Conference. His research interests include vibration control, vehicle dynamics and control systems, robust control theory and engineering applications, electric vehicles, robotics and automation, smart materials and structures. He is a recipient of the Australian Endeavour Research Fellowship (2012).

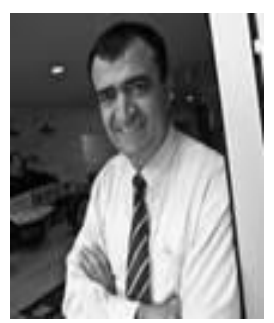

Gursel Alici received the Ph.D. degree in robotics from the Department of Engineering Science, Oxford University, Oxford, U.K., in 1994. He is currently a Senior Professor at the University of Wollongong, Wollongong, Australia, where he is the Head of the School of Mechanical, Materials, Mechatronic and Biomedical Engineering. His current research interests are soft robotics, system dynamics and control, robotic drug delivery systems, novel actuation concepts for biomechatronic applications, robotic mechanisms and manipulation systems, soft and smart actuators and sensors, and medical robotics. He has published more than 300 refereed publications in his areas of research.

Prof Alici was a Technical Editor of the IEEE/ASME TRANSACTIONS ON MECHATRONICS during 2008-2012. $\mathrm{He}$ is a Technical Editor of the IEEE Access, the first IEEE open access journal with interdisciplinary scope. He is a Member of the Mechatronics National Panel formed by the Institution of Engineers, Australia. He has served on the international program committee of numerous IEEE/ASME International Conferences on Robotics and Mechatronics. He was the General Chair of the 2013 IEEE/ASME International Conference on Advanced Intelligent Mechatronics held in Wollongong, Australia. He is the leader of Soft Robotics for Prosthetic Devices theme of the ARC Center of Excellence for Electromaterials Science. He received the Outstanding Contributions to Teaching and Learning Award in 2010 and the 2013 Vice-Chancellor's Interdisciplinary Research Excellence Award from the University of Wollongong.

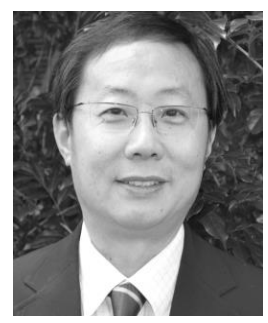

Weihua Li, PhD, is a Senior Professor and Director of the Advanced Manufacturing Research Strength at the University of Wollongong. He completed his BEng (1992), MEng (1995) at University of Science and Technology of China, and PhD (2001) at Nanyang Technological University (NTU). He was with the School of Mechanical and Aerospace Engineering of NTU as a Research Associate/Fellow from 2000 to 2003, before he joined the School of Mechanical, Materials and Mechatronic Engineering as a Lecturer. His research focuses on smart 
materials and their applications, microfluidics, rheology, and intelligent mechatronics. He is serving as editor or editorial board member for several international journals, including IEEE/ASME Transactions on Mechatronics, Smart Materials and Structures, Scientific Reports, etc. He has published more than 320 journal and conference papers. He is a recipient of
Fellow of the Engineers Australia, the Fellow of Institute of Physics (UK), Australian Endeavour Research Fellowship, JSPS Invitation Fellowship, Vice-chancellor's Award for Interdisciplinary Research Excellence, and numerous Best Paper Awards. 

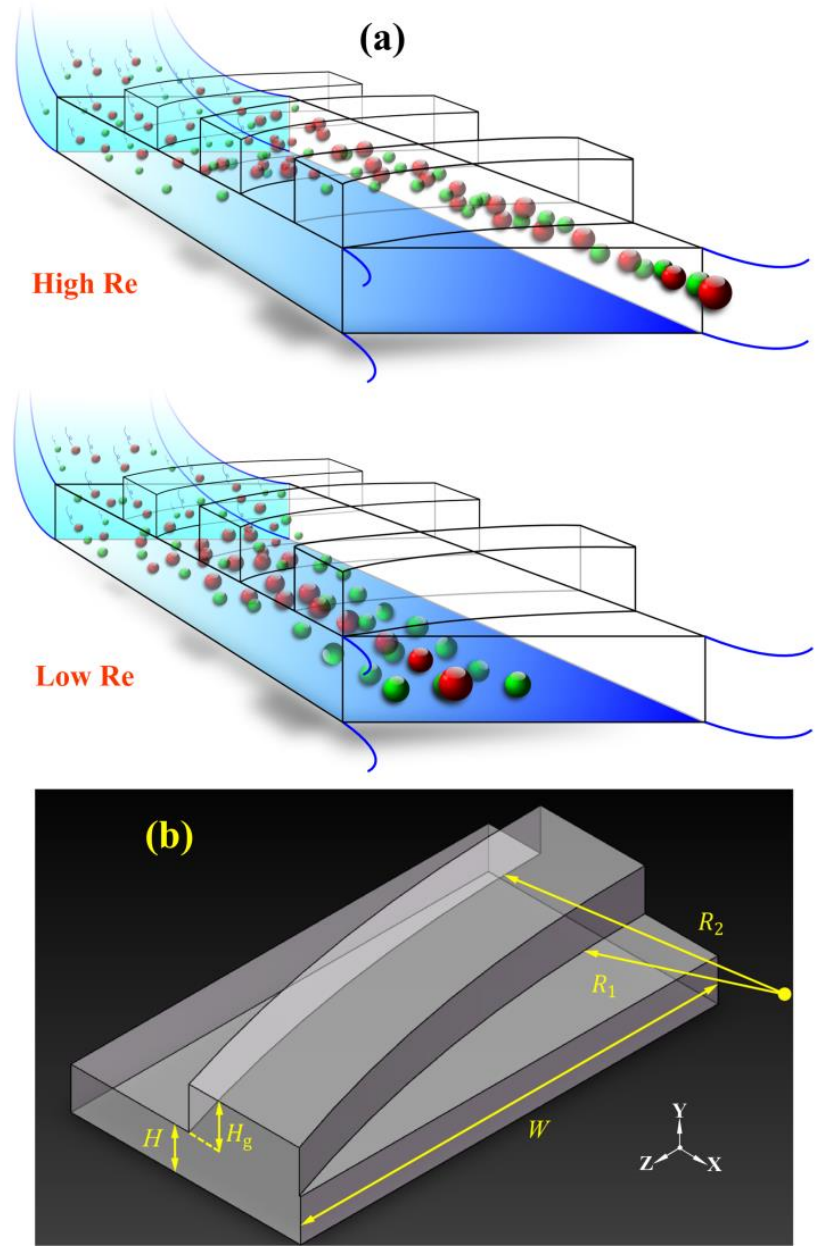

Fig. 1. (a) Schematic drawing of the double-mode microparticle manipulation in the microchannel with arc-shaped groove arrays. (b) Dimensions of the characteristic expansive arc-shaped groove structure. 
(a)

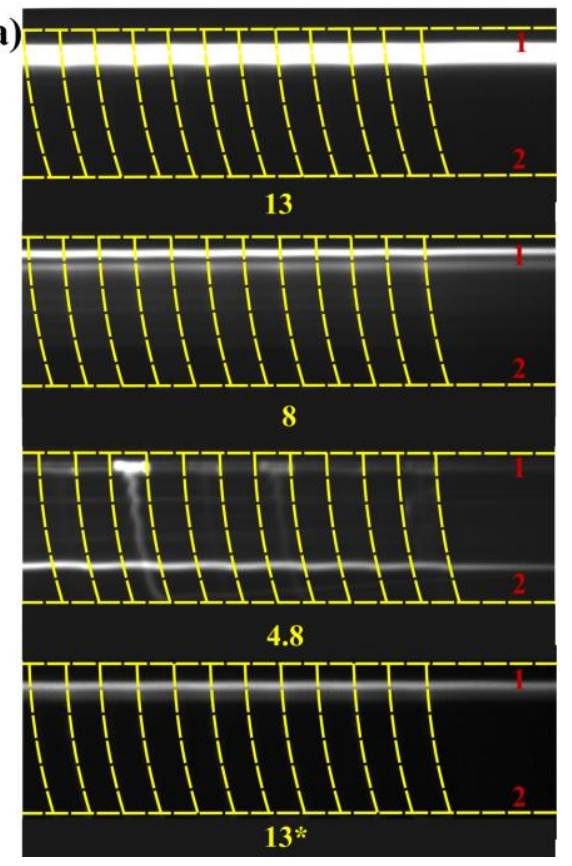

(b)
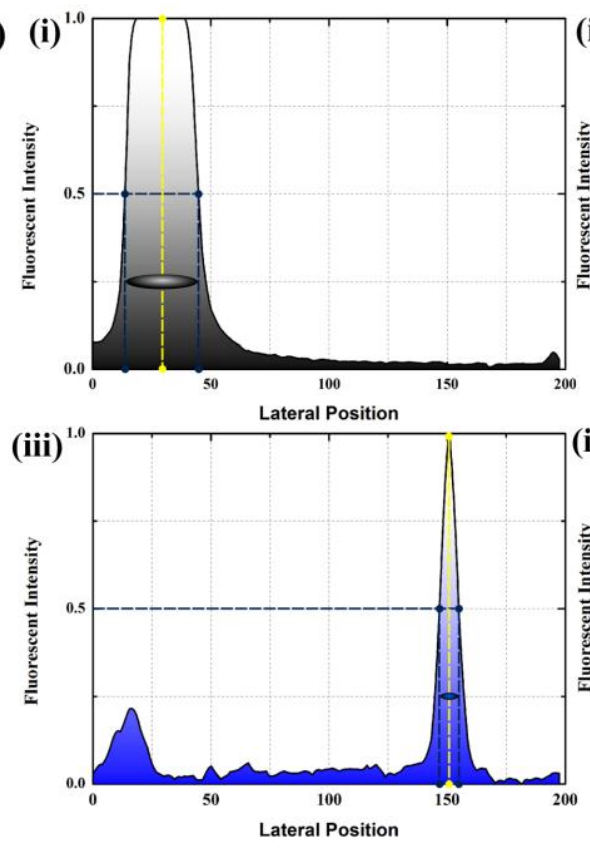
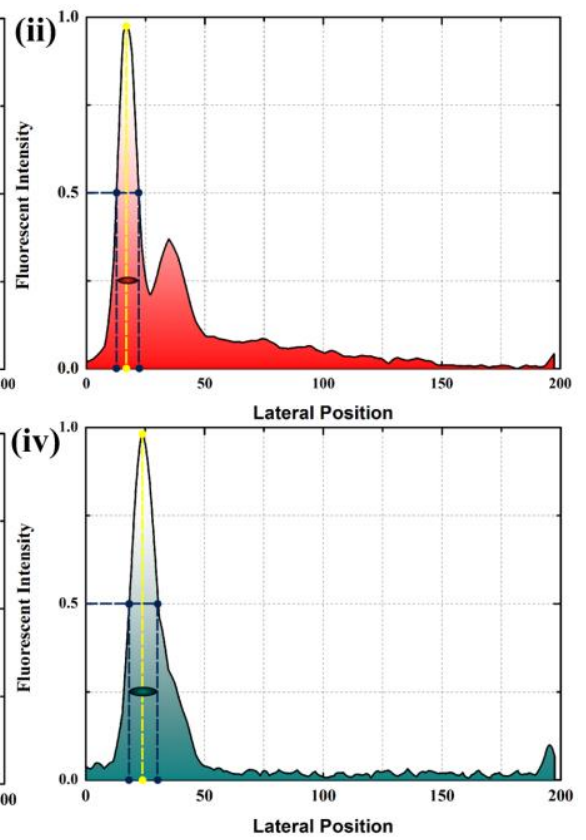

Fig. 2. Distribution of different-size microparticles at high Reynolds number. (a) Fluorescent particle trajectory patterns captured at the flow rate of $800 \mu \mathrm{l} / \mathrm{min}$ (Re is 127.27). (b) Corresponding normalized intensity profiles of different-size particle trajectory patterns. The unit of lateral position is $\mu \mathrm{m}$. (i) Normalized intensity profile of $13 \mu \mathrm{m}$; (ii) Normalized intensity profile of $8 \mu \mathrm{m}$; (iii) Normalized intensity profile of $4.8 \mu \mathrm{m}$; (iv) Normalized intensity profile of $13 \mu \mathrm{m}$ (with a low particle concentration). 
(a)

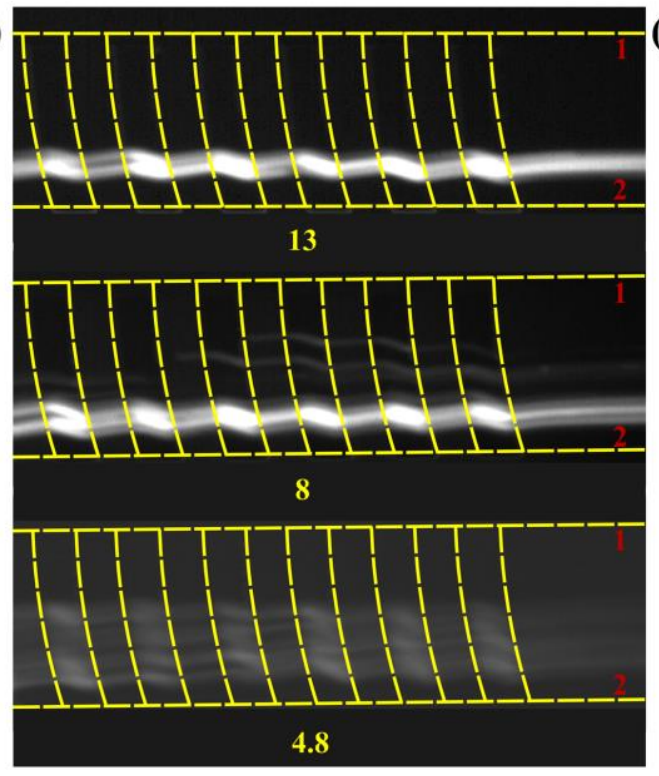

(b)
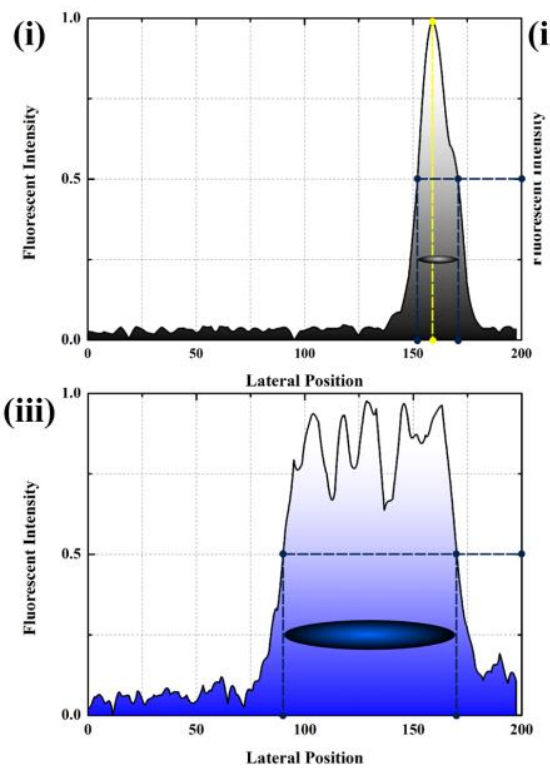

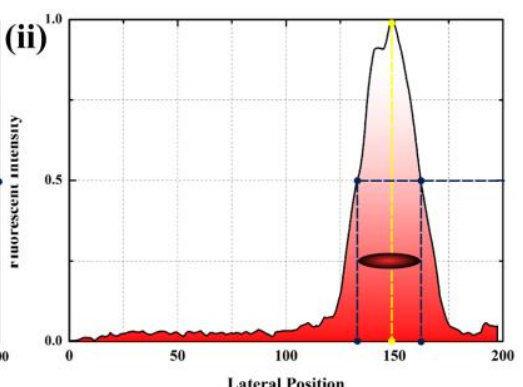

Lateral Position

Fig. 3. Distribution of different-size microparticles at low Reynolds number. (a) Fluorescent particle trajectory patterns captured at the flow rate of $15 \mu \mathrm{l} / \mathrm{min}$ ( $\operatorname{Re}$ is 2.39). (b) Corresponding normalized intensity profiles of different-size particle trajectory patterns. The unit of lateral position is $\mu \mathrm{m}$. (i) Normalized intensity profile of $13 \mu \mathrm{m}$; (ii) Normalized intensity profile of $8 \mu \mathrm{m}$; (iii) Normalized intensity profile of $4.8 \mu \mathrm{m}$. 
(a)

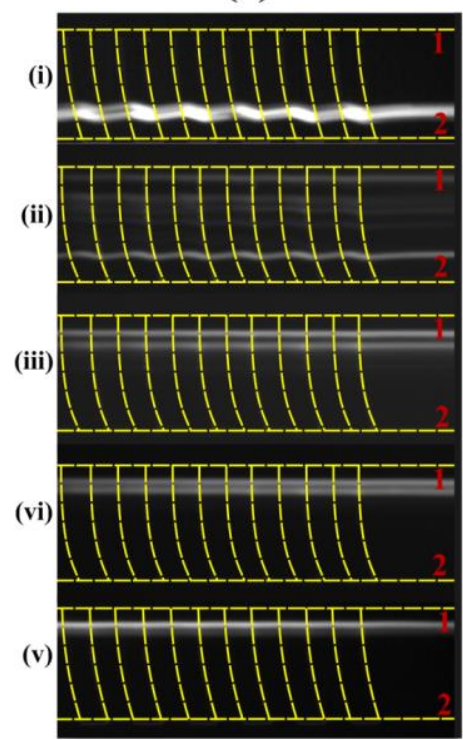

(b)

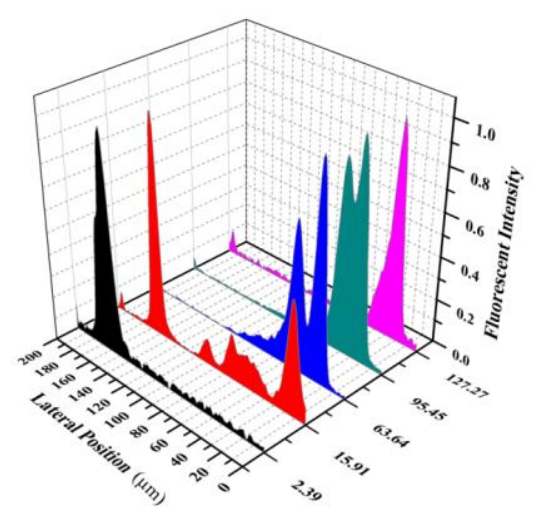

Fig. 4. $13 \mu \mathrm{m}$ particle's trajectory patterns at various Reynolds numbers (a) Fluorescent particle trajectory patterns captured at Reynolds number of (i) 2.39; (ii) 15.91; (iii) 63.64; (iv) 95.45; (v) 127.27. (b) Corresponding normalized intensity profiles of 13 $\mu \mathrm{m}$ particle's trajectory patterns. 


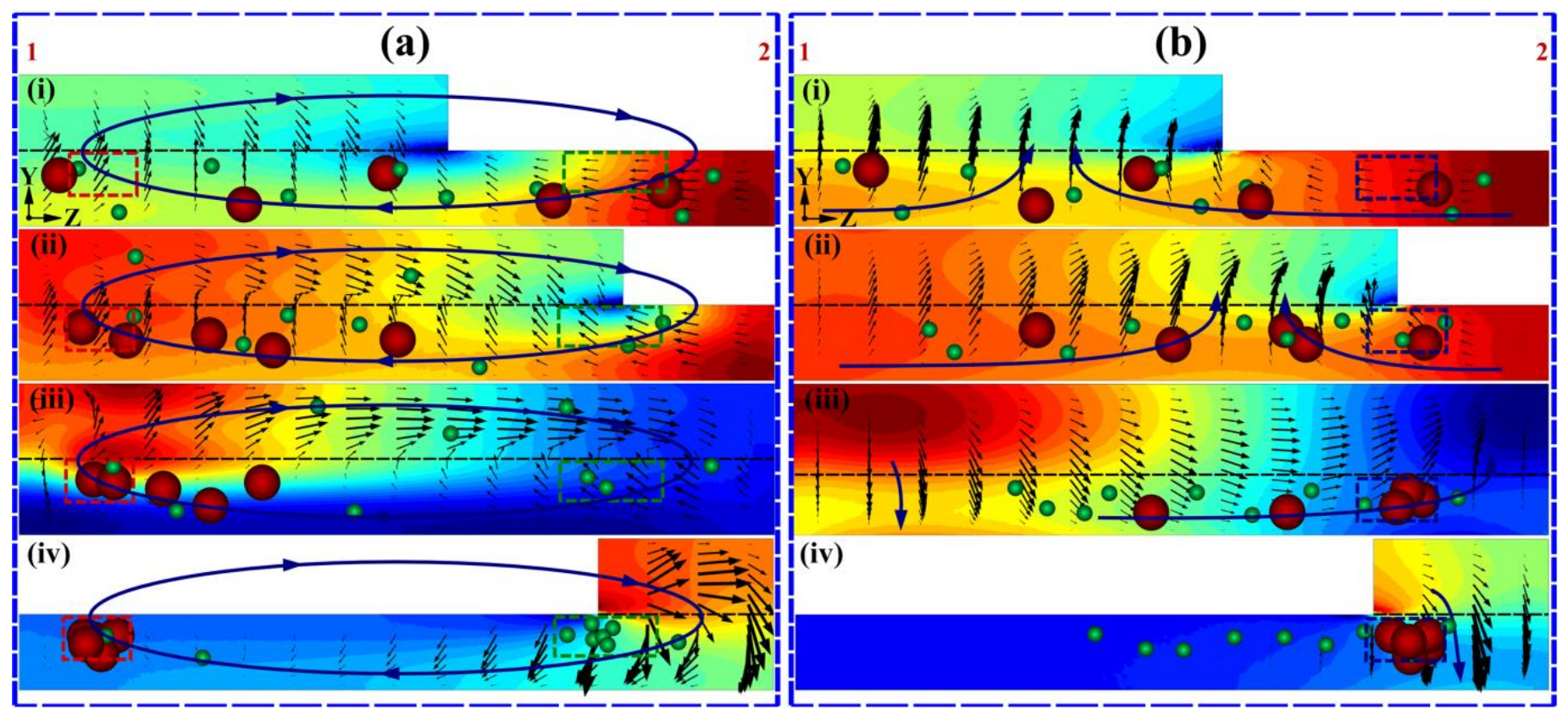

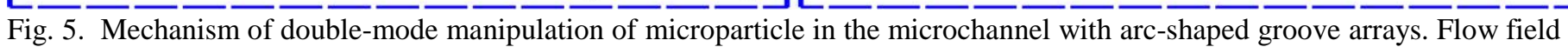
simulation achieved by COMSOL Multi-physics 5.1. (a) The simulated results at the high Reynolds number (127.27). (b) The simulated results at the low Reynolds number (2.29). (i) (iv) The cross-sectional flow field simulation plots depicted as the combination of velocity arrow and pressure contour. The arrow size is proportional to the magnitude of flow velocity. (See the unit bars in the supplementary material) 


\section{Supplementary Material for}

\section{Double-mode Microparticle Manipulation by Tunable Secondary Flow in Microchannel With Arc-shaped Groove Arrays}

Qianbin Zhao ${ }^{1}$, Sheng Yan $^{1}$, Dan Yuan ${ }^{1}$, Jun Zhang ${ }^{2 *}$, Haiping Du $^{3}$, Gursel Alici ${ }^{1}$ and Weihua $\mathrm{Li}^{1 *}$

${ }^{1}$ School of Mechanical, Materials, Mechatronic and Biomedical Engineering, University of Wollongong, Wollongong, NSW 2522, Australia, Fax: +61 24221 3238; Tel: +61 24221 3490; E-mail: weihuali@uow.edu.au

${ }^{2}$ School of Mechanical Engineering, Nanjing University of Science and Technology, Nanjing 210094, China; E-mail: junzhang@njust.edu.cn

${ }^{3}$ School of Electric, Computer and Telecommunication Engineering, University of Wollongong, Wollongong, NSW 2522, Australia 
The numerical simulation of flow field was conducted by the finite element software (COMSOL Multi-physics 5.1, Burlington, MA). For the typical microfluidic system, steady laminar flow physics was employed. The boundary condition was set as no slip and the physical property of fluid was incompressible flow. The order of finite element was set as $\mathrm{P} 1+\mathrm{P} 1$, the default setting of COMSOL Multiphysics. The 3D model of the channel was meshed with free tetrahedral grid at the default extra fine level (3017863 elements in total) to ensure the accuracy of numerical results. At the inlet, the input velocity of flow was set as 3.333 and $0.0625 \mathrm{~m} / \mathrm{s}$, respectively (the corresponding Reynolds numbers were 127.27 and 2.39). Meanwhile, the relative pressure was set as $0 \mathrm{~Pa}$ at the outlet. 


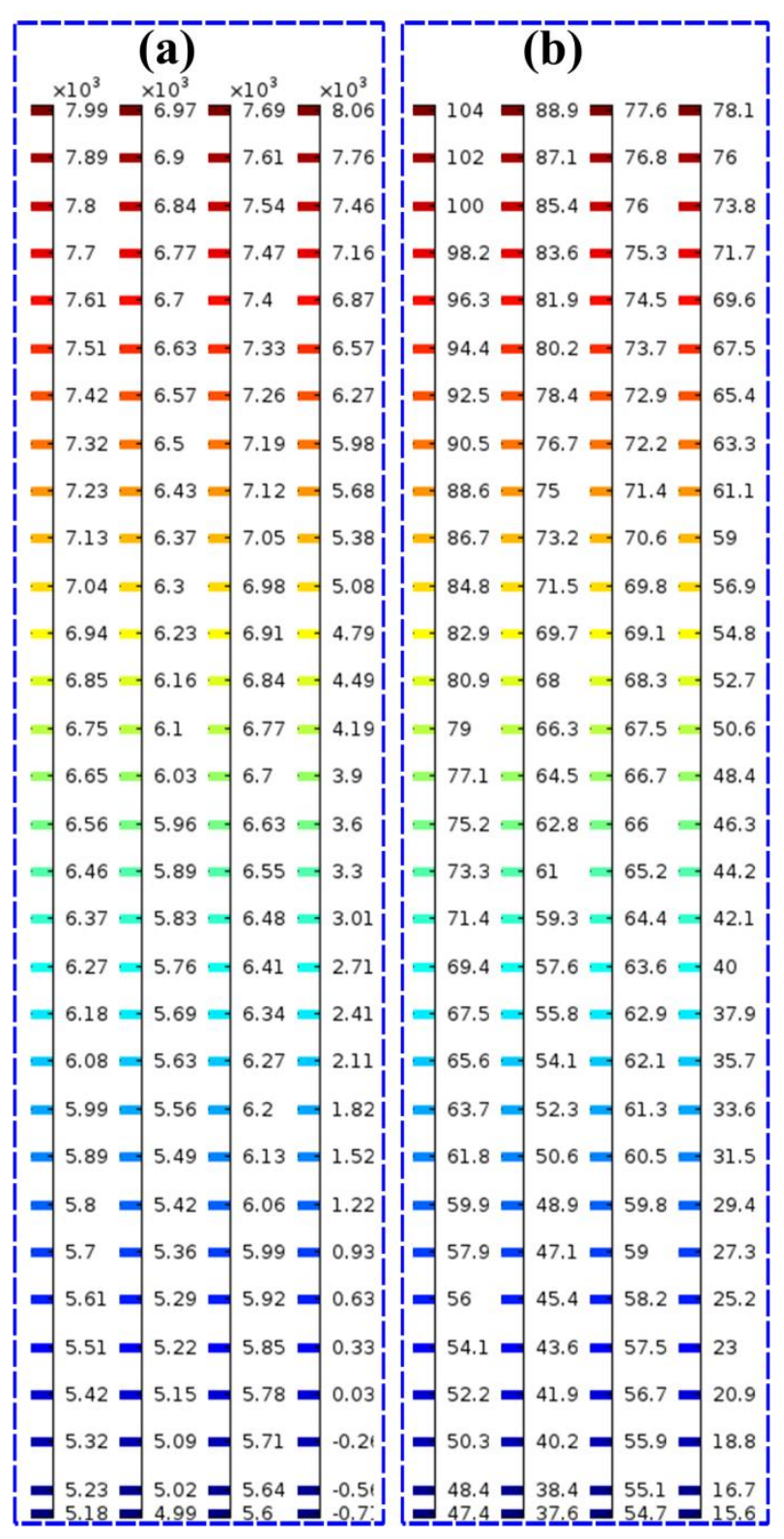

Fig. S1. Unit bars of numerical simulation results by COMSOL. (a) The simulation results at the high Reynolds number. The unit is Pa. (b) The simulation results at the low Reynolds number. The unit is Pa. 
(a)
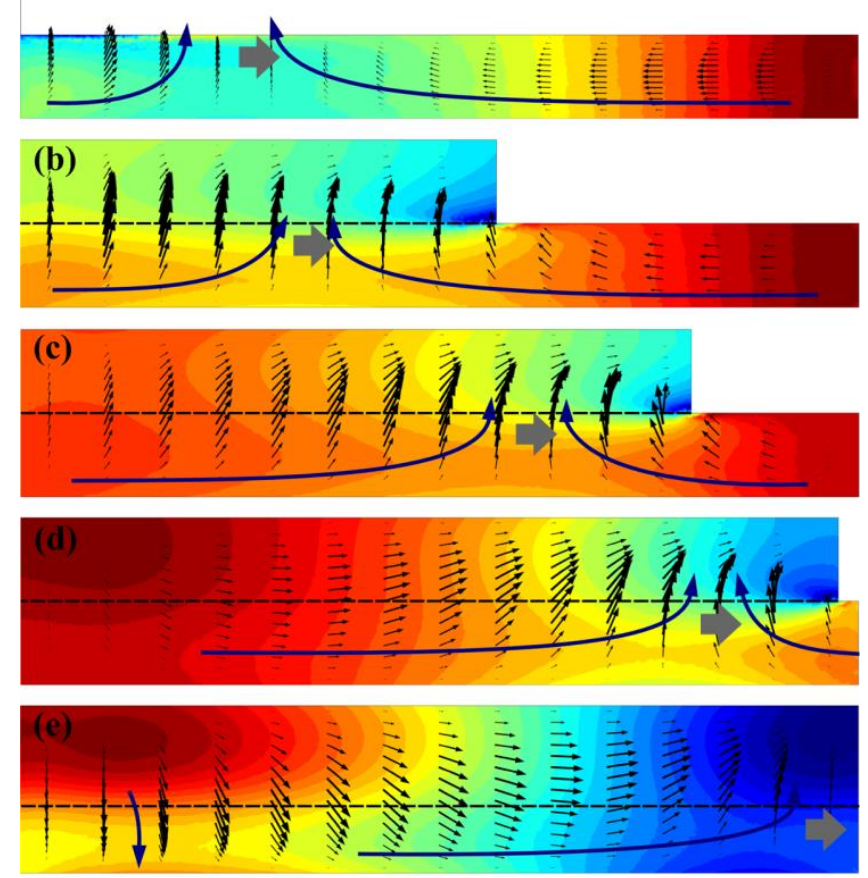

(f)

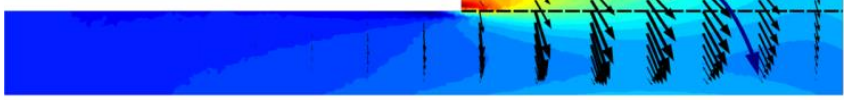

(g)

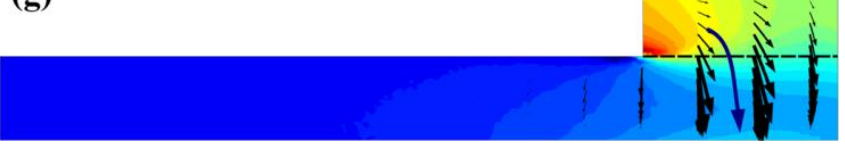

Fig. S2. The 3D model of the channel segment was divided into 7 cross-sectional plots (a-g) to illustrate the formation and development of the Dean-like vortex. The migration of the center of double microvortices is shown as the grey arrow. 